\title{
Taking DAM risk management beyond the technology: Sources of ongoing business-related risk and practical ways to address them
}

\begin{abstract}
Steve Kotrch
has been the Director of Publishing Technology for Simon \& Schuster since 1993. Charged with using technology to lower the publishing costs and time to market for products, Steve has brought the company from desktop publishing as a proof-of-concept to publishing systems as mission-critical, including not only print but web and e-books. His first foray into digital asset management was a collection of book cover images designed to enhance the sales force automation system, but which found its real value in facilitating the production of catalogs and other marketing materials. Earlier, Steve designed and managed installation of publishing systems for a wide range of clients for Electronic Directions, a consulting and training firm, the Rodale family of magazines (Prevention, Runner's World, Bicycling, Men's Health, Backpacker etc) and various book and financial publishers. He also created a publishing system for Value Line, Inc., which has been in use now for almost 20 years.
\end{abstract}

Keywords: project risk, risk avoidance, pilot project, change management, implementation failure

Abstract Normally the discussion of risk in terms of technology implementations focuses on risk associated with the technology. When it comes to digital asset management (DAM), this represents the lesser risk. The greater risk is that users will ignore the DAM system and continue organizing (or not) their digital files in the ways they always have done. The author focuses on lessons learned through three implementations of DAM at a major publishing house: on sources of risk, and on practical ways to address them.

Steve Kotrch

Director of Publishing Technology,

Simon \& Schuster.

1230 Avenue of the

Americas,

New York, NY 10020

USA

Tel: +12126987000

Email: steve.kotrch@

simonsays.com

\section{JUST BECAUSE YOU BUILD IT . . .}

This journal has already discussed one element of risk in the implementation of digital asset management (DAM). ${ }^{1}$ This is the risk involved in the technical side of implementing DAM, the software and the infrastructure. It has been my experience that while much of the discussion of DAM implementation focuses on technology, this is the smaller part of the equation. The greater risk is that the effort will be wasted.

The fact is, the business community does not care a fig about a technologist's plans or what he or she may have created. While there are no statistics on this highly embarrassing type of failure, anecdotal reports abound of expensive systems lying fallow for a variety of business-related reasons: company politics, inadequate buy-in from the business, awkward design, etc. Here we examine some of these ongoing risks, based on the author's experience through three implementations, of various sizes of DAM systems. 


\section{SYSTEM FAILURE DOES NOT SPELL DOOM}

The argument behind focusing on technological risk is that, if the technology is inadequate to all the tasks it is to perform, the system will be a failure. One is given to think that even the smallest shortfall in capability will result in disaster; fault tolerance is king. This does not square with my experience: The system can't export all 25,000 product shots? Write a SQL routine that locates them and a batch process that copies them to a DVD. An errant, illegal character in a data feed causes advanced search capabilities not to work? Identify the source and then use a searching algorithm to find the culprit. In the mean time, you may have to use multiple simple searches to get your work done, and it may result in overtime, but that isn't exactly doom.

\section{INITIAL RISK}

The risk of failure is at its highest when first implementing DAM, particularly at the enterprise level. Overcoming the resistance to change has been addressed by Dennis Pannuto in these pages. ${ }^{2}$ I believe that what is needed is, as Pannuto says, a thorough understanding of the business, and to get buy-in from the group who is managing the day-today operation of the firm - the people I think of as the influencers - as well as from upper management.

\section{SELLING THE CONCEPT}

Getting buy-in from the influencers is the same as selling anyone on anything. They respond to DAM as they would respond to any other major purchase or change. Speak to a car dealer and he will tell you that the key to making the deal is to get the customer to test drive the car. Speak to a restaurateur and you will hear that word of mouth is of vital importance.

\section{THE PILOT}

A way to get the benefit of both of these sales tools is by introducing a pilot system. The intent is to give the influencers a taste of what is to come, similar to an automobile test drive. It also creates buzz, the kind of word of mouth that can help generate interest and buy-in throughout the community. Configured in a certain way, a pilot project can also spread DAM virally through an organization.

The pilot approach provides an additional benefit. Being on a smaller scale, it allows you to tailor your implementation to the business. This not only helps you test your assumptions and come up with a more effective design, it should help convince the influencers of the seriousness of your intent.

\section{THINGS TO KEEP IN MIND}

The danger of building a pilot such as I describe is that it may become too successful. The user community may be satisfied with your sample, and may reject your vision for a more full-blown system.

Perhaps it is just common sense, but the implementer should also keep in mind that a pilot project should be treated with all seriousness in terms of the assets involved. They must be the real thing - full-resolution graphics, no lossy compression - in other words, assets that are of a quality and organized in such a way that they can be transferred directly into the fully implemented system. Ideally it should 
zero in on what Pannuto calls the "ultimate asset".

\section{AN EXAMPLE}

The enterprise-level DAM system at Simon \& Schuster is called the Digital Asset Bank (DAB). In the process of setting up the DAB, we first set up and made widely available a type of pilot, a minimally configured system that offered easy, company-wide access to only three assets: product images (typically a high-resolution image of the front cover of a book), author photos in Associate Press format, and recent catalogs in PDF format. This provided instant proof of the value of DAM.

The existence of this system was announced at a wide variety of meetings, from editorial meetings to company-wide "town halls" as well as on the intranet and in written announcements. People who used to have to order books so they could scan covers for their needs suddenly became very interested in this new resource.

\section{EXECUTIVE SUPPORT}

Executive support for the DAB was imperative for funding the project, of course, and a pilot system, as proof of concept, was instrumental in obtaining that. In our case the backing of the executive was key in establishing a certain contract between creatives designers and others who were responsible for populating the $\mathrm{DAB}$ with assets - and everyone else.

\section{THE CONTRACT}

Before the DAB, people would obtain materials they needed from creative departments: author photos, copies of book jackets and covers, etc. At an early stage of implementing the DAB we put "the contract" into effect: If anyone requests files from a creative department, they are referred to the $\mathrm{DAB}$, and the creatives have the right to refuse to provide them. By the same token, anyone requesting access to the $\mathrm{DAB}$ is promptly given a short training session and a login.

Creative departments readily accepted the contract. They agreed to take the extra effort to import assets in a timely manner, and in return they are free to concentrate on their jobs without the interruptions of having to hunt down and retrieve finished materials.

\section{ENFORCEMENT}

Success, of course, is based on the complete, accurate and timely population of the system with assets; and the risk is that the creatives will not keep up their end of the bargain. The weight of executive mandate helps, of course, but on a practical, day-to-day basis the key is accurate reporting of which assets are due to appear in the DAB and when. This allows management to monitor and follow up, and allows self-management by creatives. The last thing our creative departments want is to be called to task by those departments who are relying on timely delivery of those assets.

\section{THE FUNCTION OF TRAINING}

As previously mentioned, anyone in the company is welcome to use the DAB, provided they attend a training session first. One important function of this training, pertinent to our discussion, is to manage user expectations. It is vital to point out what the system does not or cannot do, as well as what it can. Otherwise, disenchantment with the 
system can grow to destructive proportions.

Another benefit of training in terms of reducing risk in the ongoing system is that it induces a dialog that provides feedback to the DAM support team. If conducted correctly, and followed up on, it can provide information about changing needs.

\section{MANAGING DISRUPTION, AND ONGOING RISK}

DAM is a disruptive technology. It carries ongoing risk. It creates new expectations, changes procedures, practices and even processes. Pannuto points out that a DAM system must be designed to fit the business' requirements to an exacting degree. I would like to add that as changes occur in the business, some of them in direct response to instituting a DAM system in the first place, the system must change or grow to accommodate them. Not doing so carries risk to the system. As a result, the team responsible for the continued upkeep of the DAM system must remain eternally vigilant.

People are resourceful, and this can work to the benefit of DAM in the short term, as, for example, when faced with systematic shortcomings. But if the DAM system does not change to accommodate their changing needs, these resourceful users will circumvent the system. This circumvention is a threat to even a fully established DAM system, one that has become an integral part of a firm's landscape, because it can eat away at the underpinnings of DAM as a corporate policy.

\section{THE IMPORTANCE OF FOLLOW-UP}

To keep the DAM system in tune with the business, constant monitoring and follow-up with users is necessary.

Listening to users' complaints and suggestions is of course necessary, but a proactive approach is called for.

Proactive follow-up with our publicists, for example, convinced them that, with a little training they could easily use the DAM system to eliminate the physical duplication of author photos and other materials for the media, along with the necessity of sending these materials via expensive overnight delivery or messengers. Instead, they now send the AP-standard author photos, press releases etc via email or file transfer protocol (FTP). My point is that it is all well and good to tell users what they can do with the system. To get them to use it productively, in our experience, someone must actually sit down with them and demonstrate, and teach. The result is that users will feel empowered; the DAM system will earn respect.

\section{CIRCUMVENTION}

You cannot be in all places at all times, and circumvention of the system can happen behind your back. For example, as previously mentioned, all users at Simon \& Schuster have been given access to digital representations of our products, the product images, and they have learned how to put them to good use. But the system is not so elegant and quick as it can be, so when they know that they will be using certain product images repeatedly, or even on the chance that they will, they have created collections of these images in their personal folders on network fileservers, with the images named in a way that is meaningful to them, instead of by ISBN, as they are in the DAB. 
The DAB contains approximately 25,000 of these product images. We recently discovered that our sales division - actually only the in-house sales force, a small percentage of the total sales force - had managed to create individual caches of these images, totaling 90,000 images on their fileserver, essentially creating their own ad hoc DAM.

The knee-jerk reaction among the support group is to say that the users are misusing technology, "They need to exercise some discipline." Yet the collections we examined on the file server were actually very well organized, with each file clearly labeled. This was not a lapse of "discipline" but a lapse in our making the DAM system responsive to current needs. The users are in no way to blame.

\section{THE RISKS OF A “RISK FREE” SYSTEM}

The risk, in this example of the 90,000 "rogue" images, is that users have made collections of images that are not updated, as they are in the DAB. Like it or not, it is a result of dissatisfaction with the DAB. It breaks the contract, in a way, and risks the reputation of the DAM implementation.

The current user interface to our DAM system involves multiple screens with numerous choices that need to be made or ignored. And this interface is a direct product of creating a robust, technologically "risk free" system. It contains more than 40 fields of metadata and can process files in groups, allow users to save their search criteria, catalog and annotate assets in multiple ways, and extract them using a variety of sophisticated file format transformations, and so on.
The nature of this highly secure, very sophisticated and robust DAM system makes the collection of materials tedious, and transmission of the files that much slower than the simple copying from a file server to the desktop. It is small wonder that resourceful users find alternate ways to collect and access the digital files they need.

To mitigate this risk and restore the reputation of the $\mathrm{DAB}$, it must be modified, or perhaps another means of access must be created that is quick and easy to use, for the purposes of this important group of users. We must make it function, in other words, like some of the systems we rejected when first building the DAB, as being too simple, not as robust or secure, and potentially carrying more technological risk.

To summarize, the implementation of DAM must hew closely to the needs of the business, and of the people involved in that business, in order to be useful. If it is not useful, it will not be used, and that is the greatest risk. In addition, the implementers must constantly monitor that fit, and make adjustments where necessary, in a timely manner. Merely following the textbook rules of technological risk management is not enough.

\section{References}

1. De Millo, A. (2005) "Risk management strategy: A practical guide for risk awareness and its cause and effect on project deployment", Journal of Digital Asset Management, Vol. 1, No. 2, pp. 8895.

2. Pannuto, D. (2005) “The culture of change and the ultimate asset", Journal of Digital Asset Management, Vol. 1, No. 2, pp. 81-87. 